\title{
High-Resolution Stereoscopic Surgical Display Using Parallel Integral Videography and Multi-projector
}

\author{
Hongen Liao ${ }^{1}$, Nobuhiko Hata ${ }^{2}$, Makoto Iwahara ${ }^{2}$, Susumu Nakajima ${ }^{3}$, \\ Ichiro Sakuma ${ }^{4}$, and Takeyoshi Dohi ${ }^{2}$ \\ ${ }^{1}$ Graduate School of Engineering, The University of Tokyo, Tokyo, Japan \\ ${ }^{2}$ Graduate School of Information Science and Technology, The University of Tokyo \\ ${ }^{3}$ Department of Orthopaedic Surgery, The University of Tokyo Hospital \\ ${ }^{4}$ Graduate School of Frontier Sciences, The University of Tokyo \\ \{liao,noby, iwahara\}@atre.t.u-tokyo.ac.jp, \\ \{nakajima, sakuma, dohi\}@miki.pe.u-tokyo.ac.jp
}

\begin{abstract}
This paper proposes a high-resolution stereoscopic surgical display using the integral videography (IV) and multiple projectors. IV projects a computer generated graphical object by multiple rays through "fly's eye lens", which can display geometrically accurate autostereoscopic images and reproduce motion parallax in three-dimensional (3-D) space. This technique requires neither special glasses nor sensing device to track viewer's eyes, thus being suitable for pre-operative diagnosis and intra-operative use. This paper reports the use of multiple reduction projection display system and a parallelcalculation to achieve high-resolution IV image. We evaluate the feasibility of this display by developing a 3-D CT stereoscopic image and applying it to surgical planning and intra-operative guidance. The main contribution of this paper is application and modification of medical stereoscopic technique originally developed in high-resolution multi-projector stereoscopic display system.
\end{abstract}

\section{Introduction}

Stereoscopic technique has been taking an important roll in surgery and diagnosis recently, with various modes of visualization on offer [1-3]. Among previous reported methods use polarized or shuttering glasses originally developed in augmented reality domain and applied in surgical simulation and diagnosis. However, glass-based method does not always give an observer an accurate sense of depth, as the depth perception depends on the spacing of the observer's eyes, which is not always the same as the fixed lenses. In the case of this kind of stereoscopic displays, they can observe pre-fixed 2-D images, which create quasi 3-D images with distortion. Subjectively they perceive it as 3-D, but there may be significant inaccuracies in registration. Motion parallax, an alternative stereoscopic vision, cannot be reproduced without wearing a tracking device. Consequently, systems based on binocular stereoscopic vision are not good enough for medical use.

Our previous reports [4] proposed an approach to overcome these issues of binocular stereoscopic vision by using Integral Videography (IV), which was an 
animated extension of integral photography (IP) originally proposed by M.G.Lippmann [5] in 1908. IV projects a computer-generated graphical object generated by multiple rays coming through "fly's eye lens". The detailed description of IP and IV can be found in [6,7]. We further extended our work for surgical assistance in orthopedic surgery [7] and an intra-operative navigation in the trials of clinical application [4].

Though the advantage of IV has been proven in both feasibility studies and clinical applications [4-7], one of the issues still unsolved is the limitation of pixel density, originally caused by of the inadequate resolution of currently available display devices. Thus, the motivation of this paper is to overcome these issues on limited resolution by using multiple projection display system and parallel computing for fast rendering. We evaluate the feasibility of this display by developing a 3-D CT stereoscopic image and applying it to surgical planning.

The main engineering contribution of this paper is use of parallel computing and multiple optical systems for stereoscopic IV image generation, which thought to be the most effective solution for generating high-resolution stereoscopic display. This engineering contribution deploys the clinical significance of the study that the newly proposed method enables high-resolution stereoscopic visualization (or even imageoverlay) of the three-dimensionally reconstructed MR, CT, or US images.

\section{Materials and Methods}

A schematic presentation of the multi-projector IV display is presented in Figure 1. Four major components of the display system are: (1) Parallel calculation and parallel display; (2) High-resolution multi-projection system; (3) Reduction projection technique and image calibration; (4) special image formation.

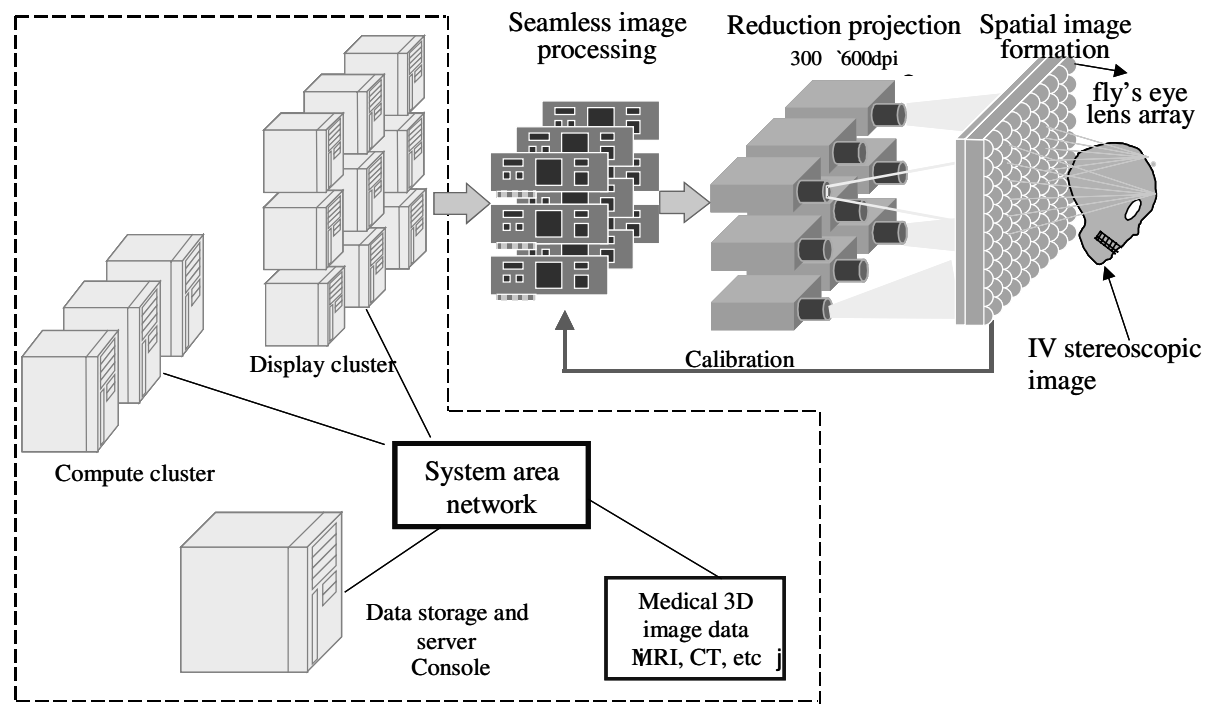

Fig. 1. Schema of the high quality IV stereoscopic display 


\subsection{Parallel Calculation and Parallel Display}

We integrated parallel calculation and display system of the IV stereoscopic display with several components (Fig.1), including 1) a display cluster with multi PCs, which are used for parallel display of high-resolution image; 2) a compute cluster with high performance computer or workstation, which was used for parallel calculation; 3) a console PC controls execution of system, including data input and storage. All the PCs are connected together with a 100 Base-T Ethernet network.

Here we face all three general types of research challenges: coordination of PCs and graphics accelerators to create consistent, real-time images; communication among multi PCs; and resource allocation to achieve good utilization. We integrated Message Passing Interface (MPI) [8] to increase the rendering performance of IV. The parallelization is based on static partitioning of the rendering image. Each node in a cluster computer is assigned a section of the line to reconstruct. The node elaborates their section in parallel and, once finished, send reconstructed section to a master process. The master process is in charge of I/O, or network communication API, which collects source images and sends the rendered image to parallel display cluster.

\subsection{High-Resolution Multi-projector Display}

Despite much recent progress in the development of new display technologies such as Organic Light Emitting Diodes (OLEDs), the current economical approach to making a large-format, high-resolution display uses an array of projectors [9]. In our system, multiple projectors of the display are arranged in display array, producing an image of high resolution across a rear projection screen by utilizing reduction projection technique, which will be described later in this paper. We wanted to maximize the number of pixels displayed, but image blending would sacrifice a large percent of pixels, especially around the four edges of each center row image.

In this case, an important issue is the coordination of multiple commodity projectors to achieve seamless edge blending and precise alignment. Seamless edge blending can remove the visible discontinuities between adjacent projectors. Edge blending techniques overlap the edges of projected, tiled images and blend the overlapped pixels to smooth the luminance and chromaticity transition from one image to another. We employed image-processing techniques to correct the source image before display by misaligned projectors. It requires only the coarsest physical alignment of the projectors.

We obtain precise alignment (or misalignment) information with an off-the-shelf camera. We zoom the camera to focus on a relatively small region of the display wall and pan the camera across the wall to get a broader coverage. The camera measures point correspondences and line matches between neighboring projectors. We then use simulated annealing to minimize alignment error globally and solve for the projection matrices.

\subsection{Reduction Projection}

Although we can achieve high-resolution image by using a normal multi-projection technique, the pixel density of projected image is not suitable for stereoscopic image creating. The pixel of the projected image used in IV stereoscopic display must be 
high-density. In this study, we use reduction projection technique achieve a highdensity pixel image. The reduction projection was usually used in the field of electron optics and exposure technique. In this study, we redesign the projector by altering the combination and arrangement of lenses to achieve new reduction projection optics. The density of pixel on the projected image can be provided over 300 dpi.

\subsection{Spatial Image Formation and Image Adjustment}

By the nature if the IV principle described, the high resolution and high-density image projected on screen must be free from distortion and reflection, so an antireflective antistatic coating [10] flat screen is used to display the image. This screen is placed in the rear of "fly's eyes lens" array. When the rendered elemental IV image is projected on the screen, the stereoscopic image will be formatted to a spatial image. In addition, we obtain precise alignment (or misalignment) information with a digital camera by measuring the position and color features of the pixels projected on a screen, and then feedback the calibration information to the image-processing hardware to make fine adjustments of the projected image on the screen.

\section{Clinical Feasibility Studies}

To evaluate the feasibility of this study, we applied this display system to image guide surgery. This kind of stereoscopic image can enhance the surgeon's capability to utilize medical 3-D imagery to decrease the invasiveness of surgical procedures and increase their accuracy and safety. These applications include surgical planning, surgical guidance, and surgical guidance with intra-operative updates. Our prior works focused on surgical guidance to present the surgeon with IV image that was gathered in pre-operative, track surgical instruments within the operating field and display in a real-time IV stereoscopic image [4]. In this study, we perform surgical planning and simulation by this newly developed IV stereoscopic display, as shown in Fig.2.

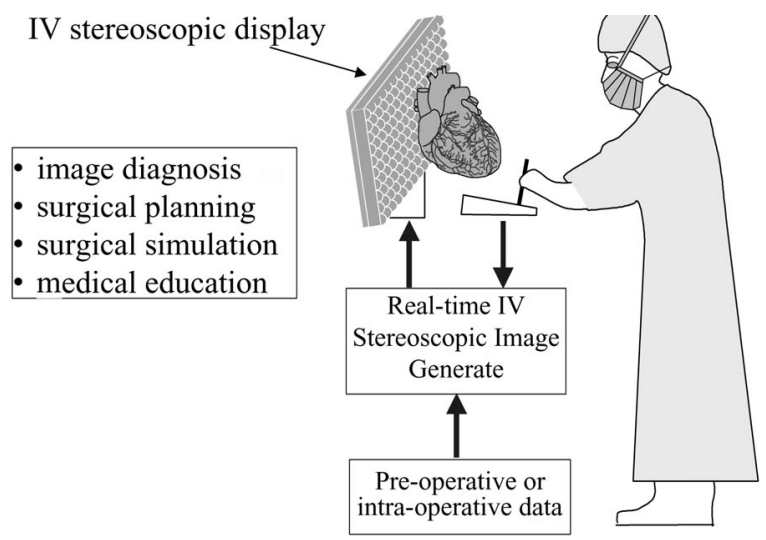

Fig. 2. Schema of surgical planning and simulation system of IV stereoscopic display 


\subsection{Equipments and Results}

We developed a multi-projector system with 9 projectors (XGA, U2-1110, PLUS Vision Corp., Tokyo Japan), arranged in a $3 \times 3$ array, which producing a display of $2872 \times 2150$ pixels across a $241 \times 181 \mathrm{~mm}$ (302.38dpi) rear projection screen by utilizing reduction projection technique. The pitch of each pixel projected on the screen is $0.084 \mathrm{~mm}$. Micro lens array placed on the screen has hexagonal micro lenses with diameter of approximately $1.008 \mathrm{~mm}$, covers 12 pixels in the reduction projection screen. The image-processing hardware (Model PA99, System Development Laboratory, Hitachi, Ltd.) was developed with the maximum pixel frequency of the $65 \mathrm{MHz}$ that corresponds to 60 frames of XGA image per second. The high-resolution multi projection systems include 9 projectors and corresponding mirror combination. This system achieves high-resolution and high-density image with reduction projection and precision position calibration. The reduction projection lenses using in this projector include 7 sets and 11 pieces of lenses. We obtain precise alignment (or misalignment) information with a camera (Nikon D1X digital camera).

In order to observe the 3-D image with correct motion parallax, one should observe at a distance of about $50 \mathrm{~cm}$ from the system. The condition wherein the projected 3D image was continuously observed was examined (Fig.3).

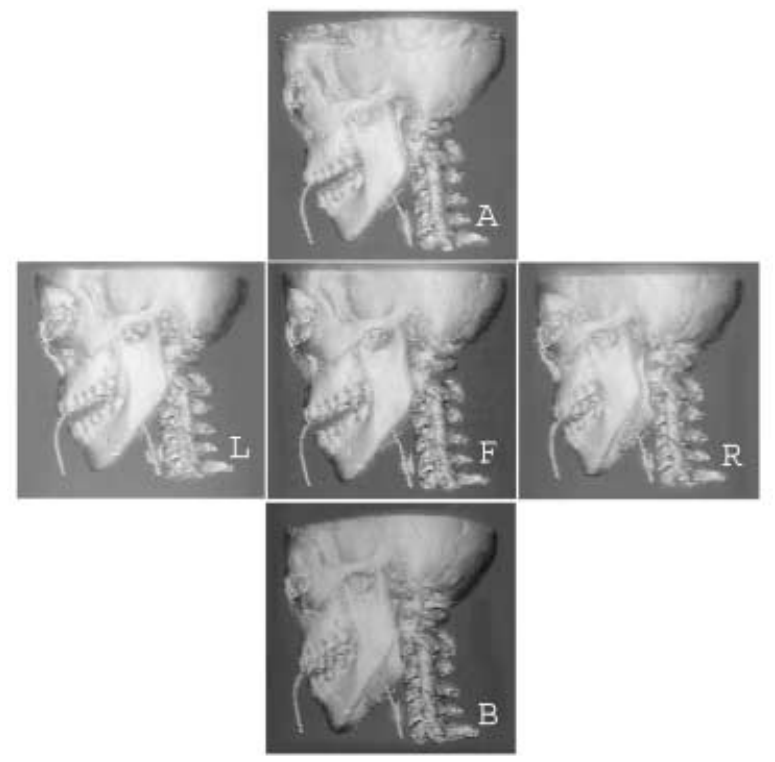

Fig. 3. Photos of projected stereoscopic skull images (stationary) by IV, taken from various directions. They show the motion parallax. The letter denotes the position of the observer to the IV stereoscopic image: A: above, L: left, F: front, R: right, B: below

\subsection{Surgical Planning and Simulation}

We evaluated the usefulness of the newly developed system in operative setting. In clinical feasibility study, we performed CT scanning to take photo of in-vivo human heart 5 times in one cardiac cycle. The volumetric CT images of human heart 
(512x512pixels x180 slices for one time, thickness of $1.0 \mathrm{~mm})$ were rendered 5 times separately in one heartbeat. The rendered elemental IV images were projected continually on the IV stereoscopic display with the same heartbeat period of the patient (Fig.4). (Since the projected IV stereoscopic image was purely threedimensional, it was difficult to record in two-dimensional conventional photographs. The quality of the actual image was much better than shown in this figure.)

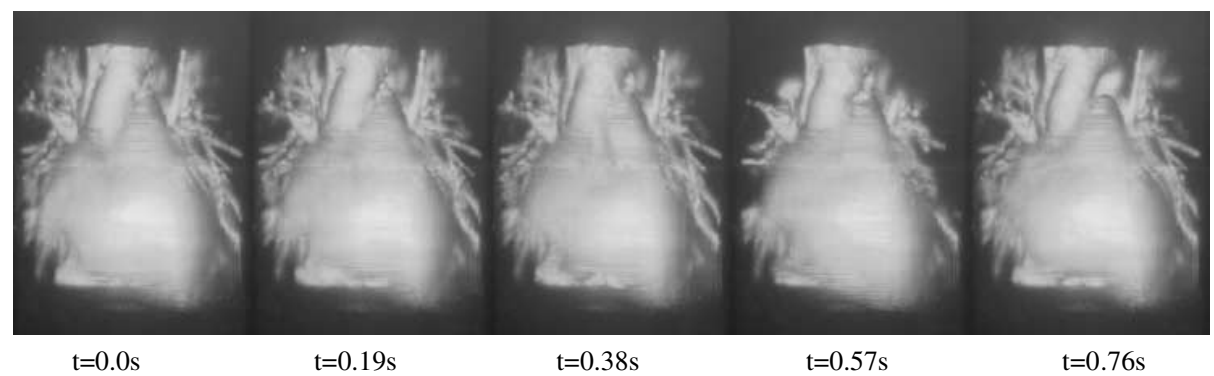

Fig. 4. IV CT stereoscopic animated image: the patient has a rate of 63 beats per minute, cardiac cycle of $0.95 \mathrm{~s}$

\subsection{Intra-operative Assistance and Guidance}

Intra-operatively, IV stereoscopic image can help with the navigation of instruments by providing a broader view of the operation field. In combination with robotic and surgical instrument, it even can supply guidance by pre-defining the path of a biopsy needle or by preventing the surgical instruments from moving into critical regions. IV image developed in this study can be segmented. Effects such as thresholding, morphological operations (dilation, erosion), island removal, cropping, and free-hand drawing can be applied to the data. One strength of IV image algorithm in our system is that effects can be visualized by multiple layers along with surgical instrument and critical regions (Fig.5).

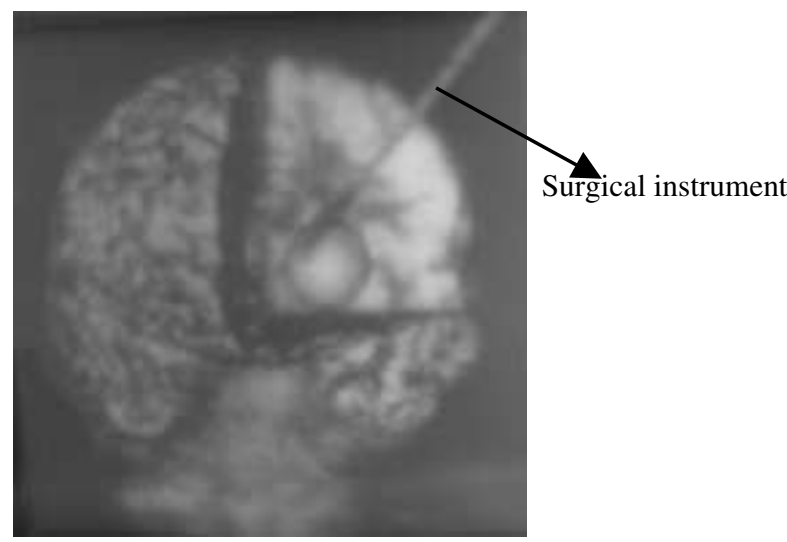

Fig. 5. Surgical simulation: IV image visualized by multiple layers along with surgical instrument and critical regions 


\section{Discussions}

The IV stereoscopic display system developed in this study has three primary merits: Superimposing the real, intuitive 3-D image for medical diagnosis and surgical guidance; Avoiding the need of extra devices such as the wearing of special glasses, and offering a geometrical accuracy image over the projected objects (esp. depth perspective); Visibility of motion parallax over a wide area, simultaneous observation by many people.

As accuracy is most important element in medical imaging, this system has advantages over other 3-D image display method. Theoretically, when the ideal lens is utilized, IV can provide a three-dimensional display that is free from any discontinuous change of images that occur with the observer's movement and with the same resolution that conventional two-dimensional displays feature [11]. The measurements of spatial resolution of IV stereoscopic display show the quality of IV image can be improved. Actually, both of the IV stereoscopic display systems developed in this study have a pixel density of only about 300dpi. The spatial resolution of the 3-D image projected is proportional to the ratio of the lens diameter in the lens array to the pixel pitch of display. Thus, both the lens diameter and the projected pixel pitch need to be made much smaller. One of the possible solutions to realize higher pixel density is the use of more multi-projectors or projector with higher resolution (SXGA or more pixels) to create more high-resolution image. A more large computation power is also needed to correspond to multi-projector system.

In the application test, we found that the display devices developed in this study is massive. The multi-projector system must be made more small and simplicity to be use in operating room, especially for the navigation use or image-overlay. This can be resolved by altering the design of projector and using the technology of the Digital Micromirror Device (DMD) directly.

\section{Conclusion}

In conclusion, we have developed a high resolution with high pixel density device for IV stereoscopic display system using multi-projector and reduction projection method. The feasibility study indicated that the multi-projector and parallel rendering performance achieved with the proposed method is satisfactory and suitable in surgical diagnosis and planning setting. We evaluate the feasibility of this display by developing a 3-D CT stereoscopic image and applying it to surgical planning. The main contribution of this paper is application and modification of stereoscopic technique originally developed in high-resolution multi-projector stereoscopic display system.

\section{Acknowledgements}

This study was partly supported by the Grant-in-Aid for the Development of Innovative Technology (12113) by the Ministry of Education, Culture, Sport, Science 
and Technology in Japan. We thank Haruo Takeda, Masami Yamasaki, Tsuyoshi Minakawa, Takafumi Koike, Fujio Tajima and Yasuyuki Momoi of the System Development Laboratory, Hitachi, Ltd, for their contribution in seamless technique in multi-projector display.

\section{References}

1. M.A.Guttman, E.R.McVeigh, "Techniques for fast stereoscopic MRI," Magnetic Resonance in Medicines, Vol.46, pp317-323, 2001.

2. M.Blackwell, C.Nikou, A.M.Digioia, T.Kanade, "An image overlay system for medical data visualization," Medical Image Analysis, Vol.4 pp.67-72, 2000.

3. Boerner R. "Three autostereoscopic $1.25 \mathrm{~m}$ diagonal real projection systems with tracking features," Proceedings of IDW, pp.835.838, 1997.

4. H.Liao, S.Nakajima, M.Iwahara, E.Kobayashi, I.Sakuma, N.Yahagi, T.Dohi, "Intraoperative Real-Time 3-D Information Display System based on Integral Videography," Medical Image Computing and Computer assisted Intervention MICCAI2001, LNCS 2208, pp.392-400, 2001.

5. M.G.Lippmann, "Epreuves reversibles donnant la sensation du relief," J. de Phys Vol.7, 4th series, pp821-825, 1908.

6. Y.Masutani et al., "Development of integral photography-based enhanced reality visualization system for surgical support," Proc. of ISCAS'95, pp16-17, 1995.

7. S.Nakajima, K.Nakamura, K.Masamune, I.Sakuma, T.Dohi, "Three-dimensional medical display with computer-generated integral photography," Computerized Medical Imaging and Graphics, 25, pp235-241, 2001.

8. Peter S.Pacheco, "Parallel Programming with MPI," Morgan Kaufmann, 1996.

9. K. Li et al., "Building and using a scalable display wall system," IEEE Computer Graphics and Applications, Vol.20, Jul/Aug, Issue 4, pp.29-37, 2000.

10. Y.Endo et al, "A study of antireflective and antistatic coating with ultrafine particles," Advances Powder Technol., Vol.7, No.2, pp.131-140, 1996.

11. H.Hoshino, F.Okano, H.Isono, I.Yuyama, "Analysis of resolution limitation of integral photography," Optical Society of America, Vol.15, No.8, pp2059-2065, 1998. 